\title{
Tidal disruption rate of stars by spinning supermassive black holes
}

\author{
Michael Kesden ${ }^{1, *}$ \\ ${ }^{1}$ Center for Cosmology and Particle Physics, Department of Physics, \\ New York University, New York, New York 10003
}

(Dated: August 2011)

\begin{abstract}
A supermassive black hole can disrupt a star when its tidal field exceeds the star's self-gravity, and can directly capture stars that cross its event horizon. For black holes with mass $M \gtrsim 10^{7} M_{\odot}$, tidal disruption of main-sequence stars occurs close enough to the event horizon that a Newtonian treatment of the tidal field is no longer valid. The fraction of stars that are directly captured is also no longer negligible. We calculate generically oriented stellar orbits in the Kerr metric, and evaluate the relativistic tidal tensor at the pericenter for those stars not directly captured by the black hole. We combine this relativistic analysis with previous calculations of how these orbits are populated to determine tidal-disruption rates for spinning black holes. We find, consistent with previous results, that black-hole spin increases the upper limit on the mass of a black hole capable of tidally disrupting solarlike stars to $\sim 7 \times 10^{8} M_{\odot}$. More quantitatively, we find that direct stellar capture reduces tidal-disruption rates by a factor $\sim 2 / 3(1 / 10)$ at $M \simeq 10^{7}\left(10^{8}\right) M_{\odot}$. The strong dependence of tidal-disruption rates on black-hole spin for $M \gtrsim 10^{8} M_{\odot}$ implies that future surveys like the Large Synoptic Survey Telescope that discover thousands of tidal-disruption events can constrain supermassive black-hole spin demographics.
\end{abstract}

\section{INTRODUCTION}

In 1943, active galactic nuclei (AGN) were discovered with emission lines Doppler broadened to widths $\gtrsim 1,000$ $\mathrm{km} / \mathrm{s}$ [1]. Twenty years later, theorists proposed that these AGN were powered by accretion onto compact objects of masses $10^{5}-10^{8} M_{\odot}[2]$. Such massive objects cannot support themselves against gravitational collapse into supermassive black holes (SBHs) [3]. SBH masses are tightly correlated with the luminosity [4, mass [5], and velocity dispersion [6] of the spheroidal component of their host galaxies.

SBHs primarily grow by accreting gas driven into galactic centers by tidal torques during major mergers [7, 8]. However, SBHs can also grow by directly capturing stars that cross their event horizons or by accreting debris from stars passing close enough to be tidally disrupted 9. Such tidal-disruption events (TDEs) could also power bright flares of radiation as the stellar debris is shock heated and subsequently accreted [10, 11. Several potential TDEs have been found in x-ray 12, UV [13, and optical [14] surveys, and tidal debris may also fuel recent blazar activity seen by the Swift satellite [15 18. The handful of TDEs found in current surveys implies that thousands more may be found each year in future surveys by the Large Synoptic Survey Telescope [14, 19]. A detailed comparison between predicted and observed TDE rates will provide important constraints on SBHs and the galactic centers in which they reside.

Frank and Rees [9] were among the first to estimate TDE rates. They introduced the concept of a "loss cone" in the stellar phase space that would be depopulated by tidal disruption within a dynamical time $t_{\mathrm{dyn}}$. Stars

*Electronic address: mhk10@nyu.edu within the loss cone have velocities lying in a cone about the radial direction. TDE rates are set by the rate at which stellar diffusion from other portions of phase space refills this loss cone. Frank and Rees evaluated stellar fluxes into the loss cone at a critical radius $r_{\text {crit }}$ at which stellar diffusion operating on a reference time scale $t_{R}[20$ could refill the loss cone within $t_{\text {dyn }}$. Cohn and Kulsrud 21 provided a more sophisticated treatment of stellar diffusion into the loss cone by numerically integrating the Fokker-Planck equation in energy-angular momentum space. More recently, Wang and Merritt 22] have revised predicted TDE rates using more realistic galactic density profiles and the observed relation between $\mathrm{SBH}$ mass and host-galaxy velocity dispersion [6].

These analyses focused on smaller SBHs for which a Newtonian treatment of tidal forces is valid and the number of directly captured stars is negligible compared to the number that are tidally disrupted. Manasse and Misner 23] introduced Fermi normal coordinates that are ideal for a relativistic treatment of the tidal tensor, and calculated this tidal tensor for radial geodesics of the Schwarzschild metric for nonspinning SBHs. Marck [24] generalized this calculation to generically oriented timelike geodesics of the Kerr metric 25] for spinning SBHs. Beloborodov et al. 26] used this tidal tensor to calculate the relativistic cross sections for tidal disruption for a range of initial orbital inclinations with respect to the SBH spin. Ivanov and Chernyakova [27] used a numerically fast Lagrangian model of a tidally disrupted star to investigate how stellar hydrodynamics affects these relativistic cross sections. In this paper, we combine a similar relativistic treatment of tidal disruption and direct capture with existing calculations of loss-cone physics to derive improved predictions of TDE rates for massive and highly spinning SBHs.

The first step in calculating TDE rates is to establish criteria for determining when a star is tidally dis- 
rupted. Most tidally disrupted stars are initially on highly eccentric or unbound orbits characterized by the distance $r$ of their pericenters from the SBH. An orderof-magnitude estimate of the maximum value of $r$ for which tidal disruption occurs can be obtained by equating the differential acceleration $G M R_{*} / r^{3}$ experienced by a star of mass $m_{*}$ and radius $R_{*}$ in the tidal field of a black hole of mass $M$ to the star's self-gravity $G m_{*} / R_{*}^{2}$. This implies that a star will be tidally disrupted when $r<r_{\mathrm{TD}} \simeq\left(M / m_{*}\right)^{1 / 3} R_{*}$. A star will be directly captured by the SBH when $r$ is less than the radius of the event horizon, which for a nonspinning $\mathrm{SBH}$ is equal to the Schwarzschild radius $r_{\mathrm{S}}=2 G M / c^{2}$. Since $r_{\mathrm{TD}} \propto M^{1 / 3}$ while $r_{\mathrm{S}} \propto M$, the ratio of tidally disrupted to directly captured stars will decrease with increasing SBH mass. Equating these two distances, we find that a $\mathrm{SBH}$ with mass $M$ greater than

$$
\begin{aligned}
M_{\max } & \simeq \frac{c^{3}}{m_{*}^{1 / 2}}\left(\frac{R_{*}}{2 G}\right)^{3 / 2} \\
& =1.1 \times 10^{8} M_{\odot}\left(\frac{m_{*}}{M_{\odot}}\right)^{-1 / 2}\left(\frac{R_{*}}{R_{\odot}}\right)^{3 / 2}
\end{aligned}
$$

should directly capture stars instead of tidally disrupting them.

Our estimate of $r_{\mathrm{TD}}$ assumed that the gravitational field of the SBH was that of a Newtonian point particle, which should only be valid for $r_{\mathrm{TD}} \gg r_{\mathrm{S}}$. One should be very suspicious of using this estimate at the event horizon, as we did when deriving $M_{\max }$ above. In a proper general-relativistic treatment, the spacetime of a spinning $\mathrm{SBH}$ is described by the Kerr metric [25]. The Kerr metric is a two-parameter family of solutions to Einstein's equation fully specified by the SBH mass $M$ and dimensionless spin $a / M<1$. Theoretical estimates of SBH spins depend sensitively on the extent to which SBHs grow by accretion or mergers. SBHs accreting from a standard thin disk [28] can attain a limiting spin $a / M \simeq 0.998$ [29] after increasing their mass by a factor $\sim \sqrt{6}[30$. The spins of SBHs formed in mergers vary greatly depending on whether the spins of the initial binary black holes become aligned with their orbital angular momentum prior to merger [31]. SBH spins can be inferred from observations of iron $\mathrm{K} \alpha$ lines in AGN $\mathrm{x}$-ray spectra 32. Large spins have been measured, such as $a / M=0.989_{-0.002}^{+0.009}$ in the Seyfert 1.2 galaxy MCG-0630-15 33, although reliable estimates are only available for a small number of systems.

Given the large sample of observed TDEs expected in the near future and the wide range of predicted $\mathrm{SBH}$ spins, it is important to determine the extent to which TDE rates depend on SBH spin. This is the primary goal of this paper. The greater the spin dependence, the more tightly observed TDEs will be able to constrain the distribution of SBH spins. In Sec. II] we review how the tidal field is calculated along timelike geodesics of the Kerr metric. In Sec. III, we describe the Monte Carlo simulations we performed to determine which stellar orbits lead to tidal disruption. We then use these simulations to calculate expected TDE rates in Sec. IV The implications of our findings are discussed in Sec. $\mathrm{V}$.

\section{TIDAL FIELDS ALONG KERR GEODESICS}

In Boyer-Lindquist coordinates [34] and units where $G=c=1$, the Kerr metric takes the form

$$
\begin{aligned}
d s^{2}= & -\left(1-\frac{2 M r}{\Sigma}\right) d t^{2}-\frac{4 M a r \sin ^{2} \theta}{\Sigma} d t d \phi+\frac{\Sigma}{\Delta} d r^{2} \\
& \left.+\Sigma d \theta^{2}+\left(r^{2}+a^{2}+\frac{2 M a^{2} r \sin ^{2} \theta}{\Sigma}\right) \sin ^{2} \theta d \phi^{2} 2\right)
\end{aligned}
$$

where $\Sigma \equiv r^{2}+a^{2} \cos ^{2} \theta$ and $\Delta \equiv r^{2}-2 M r+a^{2}$. This metric is both stationary (independent of $t$ ) and axisymmetric (independent of $\phi$ ). Massive test particles travel on timelike geodesics of the Kerr metric. Individual stars have masses $m_{*} \sim M_{\odot}$ much less than those of SBHs $\left(10^{6} M_{\odot} \lesssim M \lesssim 10^{10} M_{\odot}\right)$, and radii $R_{*} \sim R_{\odot} \simeq 7 \times 10^{10}$ $\mathrm{cm}$ less than the Schwarzschild radius

$$
r_{\mathrm{S}}=\frac{2 G M}{c^{2}}=2.95 \times 10^{11} \mathrm{~cm}\left(\frac{M}{10^{6} M_{\odot}}\right) \text {. }
$$

We can therefore consider them to be test particles for the purpose of determining their orbits. The position $(r, \theta, \phi)$ of a star as a function of proper time $\tau$ evolves according to the equations 35 .

$$
\begin{aligned}
\Sigma^{2}\left(\frac{d r}{d \tau}\right)^{2}= & {\left[E\left(r^{2}+a^{2}\right)-a L_{z}\right]^{2} } \\
& -\Delta\left[r^{2}+\left(L_{z}-a E\right)^{2}+Q\right] \\
\Sigma^{2}\left(\frac{d \theta}{d \tau}\right)^{2}= & Q-L_{z}^{2} \cot ^{2} \theta-a^{2}\left(1-E^{2}\right) \cos ^{2} \theta(4 \mathrm{~b}) \\
\Sigma\left(\frac{d \phi}{d \tau}\right)= & L_{z} \csc ^{2} \theta+\frac{2 M a r E}{\Delta}-\frac{a^{2} L_{z}}{\Delta},
\end{aligned}
$$

where the specific energy $E$, angular momentum $L_{z}$, and Carter constant $Q$ are conserved along geodesics.

Although Boyer-Lindquist coordinates reduce to flatspace spherical coordinates in the limit $r \rightarrow \infty$, the nonzero off-diagonal elements of the Kerr metric (2) imply that these coordinate vectors do not constitute an orthogonal tetrad at finite $r$. The gravitational-field gradients experienced by freely falling observers are more conveniently expressed by projecting them onto an orthonormal tetrad $\boldsymbol{\lambda}_{\mu}$ like that provided by Fermi normal coordinates [23]. This coordinate system can be used to specify points in the neighborhood of a central timelike geodesic, such as that traversed by a star orbiting a Kerr SBH. The timelike member of this tetrad $\boldsymbol{\lambda}_{0}$ is the tangent vector along the central geodesic, while the spacelike vectors $\boldsymbol{\lambda}_{i}(i=1,2,3)$ span the plane in the tangent space othogonal to $\boldsymbol{\lambda}_{0}$. The point $\left(\tau, x^{i}\right)$ in Fermi normal coordinates is reached by starting at the location 
of the star at proper time $\tau$ and moving a proper distance $R=\sqrt{\sum_{i}\left(x^{i}\right)^{2}}$ along the spacelike geodesic whose tangent vector is $\sum_{i} x^{i} \boldsymbol{\lambda}_{i}$.

In Fermi normal coordinates, the time-time component of the metric can be Taylor expanded as

$$
g_{\mu \nu} \lambda_{0}^{\mu} \lambda_{0}^{\nu}=-1-R_{0 i 0 j} x^{i} x^{j}+\ldots
$$

where $R_{\alpha \beta \gamma \delta}$ is the Riemann curvature tensor projected onto the orthonormal tetrad $\boldsymbol{\lambda}_{\mu}$. This implies that the tidal potential $\Phi_{\text {tidal }}$ experienced by a star is

$$
\Phi_{\text {tidal }}=-\frac{1}{2}\left(g_{\mu \nu} \lambda_{0}^{\mu} \lambda_{0}^{\nu}+1\right)=\frac{1}{2} C_{i j} x^{i} x^{j}+\ldots,
$$

where $C_{i j} \equiv R_{0 i 0 j}$ is the tidal tensor. Although the higher-order corrections to the tidal potential denoted by the ellipsis can sometimes be significant 36, in this paper we consider only the term quadratic in $x^{i}$. The tidal tensor $C_{i j}$ is a symmetric, traceless $3 \times 3$ matrix whose eigenvectors denote the principal axes along which the star is stretched or squeezed, and whose eigenvalues denote the extent of this stretching and squeezing.

The problem of calculating the tidal potential $\Phi_{\text {tidal }}$ thus reduces to choosing an appropriate orthonormal tetrad $\boldsymbol{\lambda}_{\mu}$ for generic Kerr geodesics and projecting the Riemann tensor onto this tetrad. This has already been accomplished for us by Marck [24, who found

$$
\begin{aligned}
C_{11}= & \left(1-3 \frac{S T\left(r^{2}-a^{2} \cos ^{2} \theta\right)}{K \Sigma^{2}} \cos ^{2} \Psi\right) I_{1} \\
& +6 a r \cos \theta \frac{S T}{K \Sigma^{2}} \cos ^{2} \Psi I_{2} \\
C_{12}= & {\left[-\operatorname{arcos} \theta(S+T) I_{1}\right.} \\
& \left.+\left(a^{2} \cos ^{2} \theta S-r^{2} T\right) I_{2}\right] 3 \frac{\sqrt{S T}}{K \Sigma^{2}} \cos \Psi \\
C_{13}= & {\left[\left(a^{2} \cos ^{2} \theta-r^{2}\right) I_{1}\right.} \\
& \left.+2 a r \cos \theta I_{2}\right] 3 \frac{S T}{K \Sigma^{2}} \cos \Psi \sin \Psi \\
C_{22}= & \left(1+3 \frac{r^{2} T^{2}-a^{2} \cos ^{2} \theta S^{2}}{K \Sigma^{2}}\right) I_{1} \\
& -6 a r \cos \theta \frac{S T}{K \Sigma^{2}} I_{2} \\
C_{23}= & {\left[-a r \cos \theta(S+T) I_{1}\right.} \\
& \left.+\left(a^{2} \cos { }^{2} \theta S-r^{2} T\right) I_{2}\right] 3 \frac{\sqrt{S T}}{K \Sigma^{2}} \sin \Psi \\
C_{33}= & \left(1-3 \frac{S T\left(r^{2}-a^{2} \cos ^{2} \theta\right)}{K \Sigma^{2}} \sin ^{2} \Psi\right) I_{1} \\
& +6 a r \cos \theta \frac{S T}{K \Sigma^{2}} \sin ^{2} \Psi I_{2},
\end{aligned}
$$

where

$$
\begin{aligned}
K & \equiv\left(L_{z}-a E\right)^{2}+Q \\
S & \equiv r^{2}+K \\
T & \equiv K-a^{2}+\cos ^{2} \theta \\
I_{1} & \equiv \frac{M r}{\Sigma^{3}}\left(r^{2}-3 a^{2} \cos ^{2} \theta\right) \\
I_{2} & \equiv \frac{M a \cos \theta}{\Sigma^{3}}\left(3 r^{2}-a^{2} \cos ^{2} \theta\right) .
\end{aligned}
$$

The angle $\Psi$ evolves along the geodesic to ensure that $\boldsymbol{\lambda}_{1}$ and $\boldsymbol{\lambda}_{3}$ are parallel propagated.

The fully general tidal tensor of Eq. (7) is intimidating, but we can gain insight by considering the tidal tensor for equatorial geodesics $(\theta=\pi / 2, Q=0)$ whose nonzero elements are

$$
\begin{aligned}
& C_{11}=\left(1-3 \frac{r^{2}+K}{r^{2}} \cos ^{2} \Psi\right) \frac{M}{r^{3}} \\
& C_{13}=-3 \frac{r^{2}+K}{r^{5}} M \cos \Psi \sin \Psi \\
& C_{22}=\left(1+3 \frac{K}{r^{2}}\right) \frac{M}{r^{3}} \\
& C_{33}=\left(1-3 \frac{r^{2}+K}{r^{2}} \sin ^{2} \Psi\right) \frac{M}{r^{3}} .
\end{aligned}
$$

The eigenvalues of this tensor are $M / r^{3}, \quad(1+$ $\left.3 K / r^{2}\right) M / r^{3}$, and $-2\left(1+3 K / 2 r^{2}\right) M / r^{3}$. Since the tidal force is

$$
F_{i}=-\nabla_{i} \Phi_{\mathrm{tidal}}=-C_{i j} x^{j},
$$

the positive eigenvalues correspond to directions in which the star is squeezed while the negative eigenvalues correspond to the direction in which it is streched. In the Newtonian limit $K / r^{2} \rightarrow 0$, the eigenvalues reduce to $-2 M / r^{3}$ and the doubly degenerate eigenvalue $M / r^{3}$. This degeneracy reflects the restoration of symmetry between the $\theta$ and $\phi$ directions at large $r$, where the effects of the SBH's spin are negligible. Stretching occurs in the radial direction corresponding to the eigenvalue $-2 M / r^{3}$. Note that despite one's possible intuition to the contrary, the tidal force remains finite at both the innermost stable circular orbit and even the event horizon itself.

To determine whether a star on a given orbit is tidally disrupted, we check at the pericenter of that orbit whether the outward tidal force in the direction corresponding to the negative eigenvalue of the tidal tensor exceeds the inwards Newtonian self-gravity of the star. We assume that the tidal field is maximized at the pericenter as in the Newtonian limit. If $\beta_{-}$denotes the numerical value of this eigenvalue, tidal disruption occurs if

$$
r<r_{\mathrm{TD}}=\left[\left(\frac{\left|\beta_{-}\right|}{M / r^{3}}\right)\left(\frac{M}{m_{*}}\right)\right]^{1 / 3} R_{*} .
$$


In the Newtonian limit $\beta_{-}=-2 M / r^{3}$ discussed above, this condition is equivalent to the more familiar expression

$$
r<r_{\mathrm{TD}}=\left(\frac{2 M}{m_{*}}\right)^{1 / 3} R_{*} .
$$

Although our condition (11) for tidal disruption is only approximate, we expect it to be conservative for several reasons. It neglects that the tidal force has already raised bulges on the star's surface before the star reaches the pericenter, so the stellar radius $R_{*}$ appearing in Eq. (11) should exceed its value in hydrostatic equilibrium far from the SBH. It also assumes that the star is nonrotating, while in reality the torques exerted on the tidally distorted star will cause it to partially corotate with its orbit. These torques are likely to be complicated for a generic nonequatorial Kerr geodesic, but we can gain some insight by again considering the Newtonian limit. Stars rotating with angular velocity $\Omega$ will be disrupted at a radius

$$
r<r_{\mathrm{TD}}(\Omega)=\left[\left(2+\frac{\Omega^{2} r^{3}}{G M}\right)\left(\frac{M}{m_{*}}\right)\right]^{1 / 3} R_{*}
$$

in this limit. For corotating stars on circular orbits $\left(\Omega^{2}=\right.$ $G M / r^{3}$ ), the first factor in parentheses on the right-hand side of Eq. (13) equals 3 as in the definition of the radius of the Hill's sphere [37. For a star corotating at the pericenter of a parabolic orbit like that expected for a star approaching a SBH from a large distance, this factor equals 4. Our assumption that the star is nonrotating is conservative because the condition (13) is most restrictive for $\Omega=0$, although $r_{\mathrm{TD}}(\Omega)$ only varies by the modest factor $2^{1 / 3}$.

Our criterion (11) might overestimate the rate at which stars are fully disrupted since they might lose their outer layers while maintaining their dense cores. In the Newtonian limit, Phinney [38] showed that stars will not be fully disrupted until

$$
r<r_{\mathrm{TD}}=\left(\frac{k}{f}\right)^{1 / 6}\left(\frac{M}{m_{*}}\right)^{1 / 3} R_{*},
$$

where $k$ is the constant of apsidal motion and $f G m_{*}^{2} / R_{*}$ is the star's binding energy. The factor $k / f=0.3(0.02)$ for stars with convective (radiative) atmospheres, but the exponent of $1 / 6$ ensures that $r_{\mathrm{TD}}$ is only weakly dependent on this factor. We ignore this factor and keep our criterion (11) for the remainder of this paper, but the Monte Carlo simulations described in the next section could easily be evaluated with a new criterion that incorporates this factor or a different choice of stellar properties than $m_{*}=M_{\odot}, R_{*}=R_{\odot}$.

A detailed study of the fraction of mass loss as a function of SBH and orbital parameters is beyond the scope of this paper, but such a study for selected orbital inclinations has been performed by Ivanov and Chernyakova 27. They recognized that for a given SBH mass and

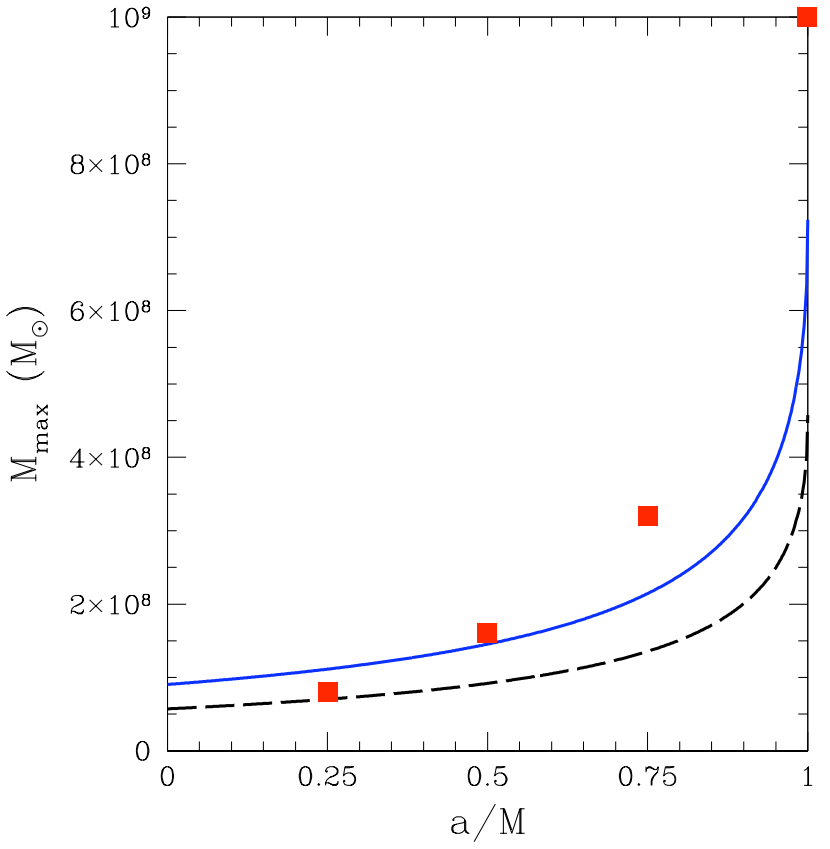

FIG. 1: The mass $M_{\max }$ of the heaviest SBH capable of disrupting a star of solar mass and radius as a function of $\mathrm{SBH}$ spin $a / M$. The red squares show the values listed in Table 2 of 27] derived using a simple hydrodynamical model. The solid blue curve shows our prediction according to the relativistic criterion (11), while the dashed black curve shows the Newtonian prediction 12 .

spin, stars on prograde, equatorial, marginally bound orbits [39] are the most likely to be tidally disrupted. Using a simple but computationally inexpensive hydrodynamical model, they calculated the mass $M_{\max }$ of the heaviest SBH capable of tidally disrupting stars without directly capturing them. As in the Newtonian prediction of Eq. 11), $M_{\max } \propto m_{*}^{-1 / 2} R_{*}^{3 / 2}$. In Fig. 1, we compare their predictions (red squares) to our own using the relativistic criterion 11) (solid blue curve) and Newtonian criterion (12) (dashed black curve) for stellar mass $m_{*}=M_{\odot}$ and radius $R_{*}=R_{\odot}$. We see that the relativistic correction to the Newtonian prediction is significant, and that our simple criterion (11) does a reasonable job given the $\sim 50 \%$ uncertainty in the simulations 27 .

We see that in the maximally spinning limit $(a / M \rightarrow$ 1 ), a SBH as massive as $\sim 10^{9} M_{\odot}$ is capable of tidally disrupting main-sequence stars. This prediction is consistent with earlier simulations [40, 41] that demonstrated this possibility. The above scaling of $M_{\max }$ with stellar mass and radius suggests that a white dwarf with $m_{*} \simeq M_{\odot}, R_{*} \simeq 0.01 R_{\odot}$ could be tidally disrupted by a maximally spinning $\mathrm{SBH}$ as massive as $10^{6} M_{\odot}$. This conclusion helps alleviate tension between the small SBH mass required for the interpretation of Swift J1644+57 as a white-dwarf tidal disruption 42 and the larger value of $M$ inferred from the relation between SBH mass and 


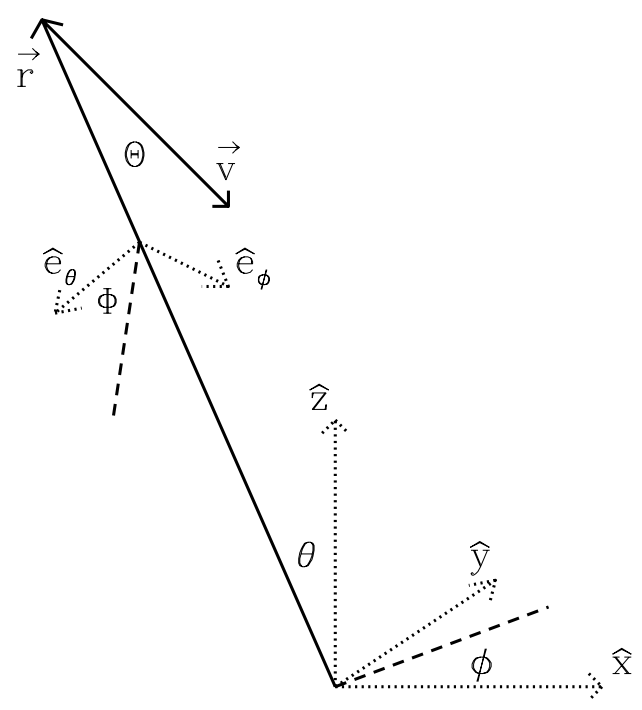

FIG. 2: Our choice of coordinates for determining the initial conditions for integrating the equations of motion (4) for stellar orbits. The SBH is located at the origin, and the star is located at Boyer-Lindquist coordinates $(r, \theta, \phi) . \Theta$ is the angle between the stellar velocity $\mathbf{v}$ and the inwards radial direction $-\hat{\mathbf{r}}$, while $\Phi$ is the angle between the component of $\mathbf{v}$ perpendicular to $\hat{\mathbf{r}}$ and the unit vector $\hat{\mathbf{e}}_{\theta}$ in the $\theta$ direction.

host-galaxy velocity dispersion [6].

\section{MONTE CARLO SIMULATIONS}

Unlike the Newtonian two-body problem, there is no general analytic solution to the relativistic equations of motion (4). We must integrate these equations explicitly for every orbit we consider. Stars that will eventually be tidally disrupted are scattered onto their final orbits at radii $r \gg r_{\mathrm{TD}}$. These orbits may or may not be gravitationally bound to the SBH, but their Newtonian orbital energies $\sim m_{*} \sigma^{2}$, where $\sigma$ is a typical velocity at $r \gg r_{\mathrm{TD}}$, are much less than the rest-mass energy $m_{*} c^{2}$. It is therefore an excellent approximation to set the specific energy $E$ appearing in Eqs. (4) equal to unity in units where $c=1$. The Kerr metric (2) is axisymmetric, so our results are independent of the initial value of $\phi$. We must perform Monte Carlo simulations with an appropriate distribution of the remaining variables $\left\{r, \theta, L_{z}, Q\right\}$ to determine what fraction of orbits are tidally disrupted according to our relativistic criterion (11), where the negative eigenvalue $\beta_{-}$of the tidal tensor $C_{i j}$ depends on all these variables.

We illustrate the geometry of the problem and our choice of coordinates in Fig. 2. We begin integrating the equations of motion (4) with the star located at an initial position $(r, \theta, \phi)$ in Boyer-Lindquist coordinates. Since these equations are independent of $\phi$ as is the tidal tensor $C_{i j}$, we do not actually need to integrate Eq. 4c. We choose an initial radius $r=2000 M$, where relativisic corrections are small, and check that our results are insensitive to this choice. In this limit, the constants of motion are given by

$$
\begin{aligned}
L_{z} & =r v \sin \theta \sin \Theta \sin \Phi \\
Q & =L_{x}^{2}+L_{y}^{2} \\
& =(r v \sin \Theta)^{2}\left(\cos ^{2} \Phi+\cos ^{2} \theta \sin ^{2} \Phi\right),
\end{aligned}
$$

where $v=(2 M / r)^{1 / 2}$ is the magnitude of the initial stellar velocity and $\Theta$ and $\Phi$ are the angles described in Fig. 2. Since the stars at $r \gg r_{\mathrm{TD}}$ do not know about the direction of the SBH spin, the stellar distribution is axisymmetric about $\hat{\mathbf{r}}$ and there is a uniform distribution in $\Phi$. Although astrophysical spheroids do not necessarily have isotropic velocity dispersions at large $r$, the stars approaching this close to the SBH belong to the tiny fraction of the distribution where the velocity lies in a loss cone centered about the radial direction [9]. Since there is no reason to expect the distribution function to be varying strongly in this small portion of phase space, there is a uniform distribution in $-1 \leq \cos \Theta \leq 1$ as well. However, since the rate at which stars of velocity $\mathbf{v}$ enter the sphere of radius $r$ is proportional to $\mathbf{v} \cdot \hat{\mathbf{r}}$, we weight our distribution by $\cos \Theta$ during our Monte Carlo simulations of stellar orbits. We choose a maximum value $\Theta_{\max }$ to avoid wasting computational time on orbits that do not closely approach the SBH.

With this choice of initial conditions, we integrate the equations of motion (4) until the star reaches the pericenter. We then calculate and tabulate the negative eigenvalue $\beta_{-}$of the tidal tensor $C_{i j}(7)$. We also tabulate which stars are directly captured by the SBH when their orbits encounter the SBH's event horizon. We integrated 250,000 stellar orbits for each of several SBH spins, with an additional 250,000 with a smaller choice of $\Theta_{\max }$ to increase our sampling of the small number of orbits that lead to tidal disruption when $M \rightarrow M_{\max }$.

\section{TDE RATES}

Given a stellar phase-space distribution function, it is reasonably straightforward to calculate the rate of TDEs using the Monte Carlo simulations described in the previous section. In Sec. IVA below, we calculate the TDE rate as a function of SBH mass assuming that the stars approach a Maxwellian distribution with fixed number density and velocity dispersion far from the SBH. This calculation illustrates the dependence of TDE rates on SBH spin. However, astrophysical SBHs reside in galaxy spheroids whose properties are tightly correlated with SBH mass 4 6]. In addition, the very luminous earlytype galaxies that host the most massive SBHs have cored 
profiles at their centers unlike the power-law profiles that characterize less luminous early-type galaxies and latetype bulges [43]. Predicted TDE rates are sensitive to whether galactic centers are described by cored or powerlaw profiles [22]. Recent observations [44] suggest that even the nuclear star cluster at our own Galactic center, long believed to have a cuspy profile $\left(\rho \propto r^{-7 / 4}\right)$ [45, may in fact have a core of radius $r_{\text {core }} \simeq 0.5 \mathrm{pc}$ [46]. Given these uncertainties, it is difficult to make precise estimates of astrophysical TDE rates. Despite this, in Sec. IVB we calculate the TDE rate assuming galaxies have isothermal $\left(\rho \propto r^{-2}\right)$ profiles at their centers and host SBHs with masses correlated with their velocity dispersions. The results of this calculation shown in Fig. 4 illustrate how SBH spin affects TDE rates.

\section{A. Maxwellian distribution}

Assume that stars far from the SBH have a Maxwellian distribution function

$$
f(\mathbf{r}, \mathbf{v})=\frac{n}{\left(2 \pi \sigma^{2}\right)^{3 / 2}} e^{-\mathbf{v}^{2} / 2 \sigma^{2}}
$$

with number density $n$ and velocity dispersion $\sigma$. The differential rate at which stars with Newtonian specific energy $E_{N}$ and angular momentum $L_{N}$ enter a sphere of radius $r$ is given by

$$
\frac{\partial^{2} \Gamma}{\partial E_{N} \partial L_{N}}=4 \pi r^{2} \int d^{3} \mathbf{v} v_{z} \delta\left(E^{\prime}-E_{N}\right) \delta\left(L^{\prime}-L_{N}\right) f(\mathbf{r}, \mathbf{v}),
$$

where the volume integral extends over the region $v_{z}>0$ and $E^{\prime}$ and $L^{\prime}$ are given by

$$
\begin{aligned}
E^{\prime} & =\frac{1}{2} v^{2}, \\
L^{\prime} & =|\mathbf{r} \times \mathbf{v}| .
\end{aligned}
$$

We can use Eq. (16) and the delta functions to evaluate the integral to find

$$
\frac{\partial^{2} \Gamma}{\partial E_{N} \partial L_{N}}=\frac{(8 \pi)^{1 / 2} n L_{N}}{\sigma^{3}} e^{-E_{N} / \sigma^{2}} .
$$

If $\sigma \ll c$, orbits near the SBH will be insensitive to the value of $E_{N}$ and we can integrate over this variable to yield a differential rate

$$
\frac{\partial \Gamma}{\partial L_{N}}=\int_{0}^{\infty} \frac{\partial^{2} \Gamma}{\partial E_{N} \partial L_{N}} d E_{N}=\frac{(8 \pi)^{1 / 2} n L_{N}}{\sigma} .
$$

The divergence of this rate as $\sigma \rightarrow 0$ results from gravitational focusing, which would channel all stars into the $\mathrm{SBH}$ in the absence of tangential velocities.

Before proceeding to the relativistic calculation, let us review the Newtonian predictions. Although the event horizon is fundamentally a relativistic concept, the "Newtonian" prediction would be that a star is directly captured by the SBH if the pericenter of its orbit is less than the Schwarzschild radius (3). The pericenter of a parabolic $\left(E_{N}=0\right)$ orbit with specific angular momentum $L_{N}$ is $L_{N}^{2} / 2 G M$. Equating this to the Schwarzschild radius, a star is directly captured if $L_{N} \leq L_{\text {cap }} \equiv 2 G M / c$, which according to Eq. 20 implies a capture rate

$$
\begin{aligned}
\Gamma_{\text {cap }}= & \int_{0}^{L_{\text {cap }}} \frac{\partial \Gamma}{\partial L_{N}} d L_{N}=\frac{(32 \pi)^{1 / 2} n(G M)^{2}}{\sigma c^{2}} \\
= & 2.1 \times 10^{-6} \mathrm{yr}^{-1}\left(\frac{M}{10^{6} M_{\odot}}\right)^{2}\left(\frac{n}{10^{5} \mathrm{pc}^{-3}}\right) \\
& \times\left(\frac{\sigma}{100 \mathrm{~km} / \mathrm{s}}\right)^{-1}
\end{aligned}
$$

A star will be tidally disrupted if the pericenter of its orbit is less than the tidal-disruption radius $r_{\mathrm{TD}}$, 12, which implies an angular momentum

$$
L_{N} \leq L_{\mathrm{TD}} \equiv\left(\frac{(2 M)^{4 / 3} G R_{*}}{m_{*}^{1 / 3}}\right)^{1 / 2}
$$

This implies a TDE rate

$$
\begin{aligned}
\Gamma_{\mathrm{TD}}^{\prime}= & \int_{0}^{L_{\mathrm{TD}}} \frac{\partial \Gamma}{\partial L_{N}} d L_{N}=\frac{(8 \pi)^{1 / 2} n G M R_{*}}{\sigma}\left(\frac{2 M}{m_{*}}\right)^{1 / 3} \\
= & 6.3 \times 10^{-5} \mathrm{yr}^{-1}\left(\frac{M}{10^{6} M_{\odot}}\right)^{4 / 3}\left(\frac{n}{10^{5} \mathrm{pc}^{-3}}\right) \\
& \times\left(\frac{\sigma}{100 \mathrm{~km} / \mathrm{s}}\right)^{-1}
\end{aligned}
$$

This rate agrees with that in Eq. (16b) of Frank and Rees [9] which applies when the critical radius at which the loss cone refills on a dynamical time exceeds the SBH's radius of influence. If TDEs can only be observed when the tidal debris is not directly captured by the $\mathrm{SBH}$, the observed TDE rate will be $\Gamma_{\mathrm{TD}}=\Gamma_{\mathrm{TD}}^{\prime}-\Gamma_{\text {cap }}$. Since $\Gamma_{\mathrm{TD}}^{\prime} \propto M^{4 / 3}$ while $\Gamma_{\text {cap }} \propto M^{2}$, the TDE rate will vanish for $M \geq M_{\max }$ (1) at which $r_{\mathrm{TD}}=r_{s}$.

We can use this same differential rate $\partial \Gamma / \partial L_{N}(20)$ to calculate the relativistic direct-capture and TDE rates. However, we must now rely on the Monte Carlo simulations of Sec. III to determine whether stars are directly captured or tidally disrupted, instead of the simple Newtonian expressions for $L_{\text {cap }}$ and $L_{\mathrm{TD}}$ given above. The simulated orbits have a maximum angular momentum $L_{\max } \equiv(2 G M r)^{1 / 2} \sin \Theta_{\max }$. The total rate at which stars on these orbits enter a sphere of radius $r=2000 M$ is

$$
\Gamma_{\text {tot }}=\int_{0}^{L_{\max }} \frac{\partial \Gamma}{\partial L_{N}} d L_{N}=\frac{(8 \pi)^{1 / 2} n G M r}{\sigma} \sin ^{2} \Theta_{\max } .
$$

The rate $\Gamma_{\text {cap }}$ at which stars are directly captured by the $\mathrm{SBH}$ is found by multiplying this total rate $\Gamma_{\text {tot }}$ by 


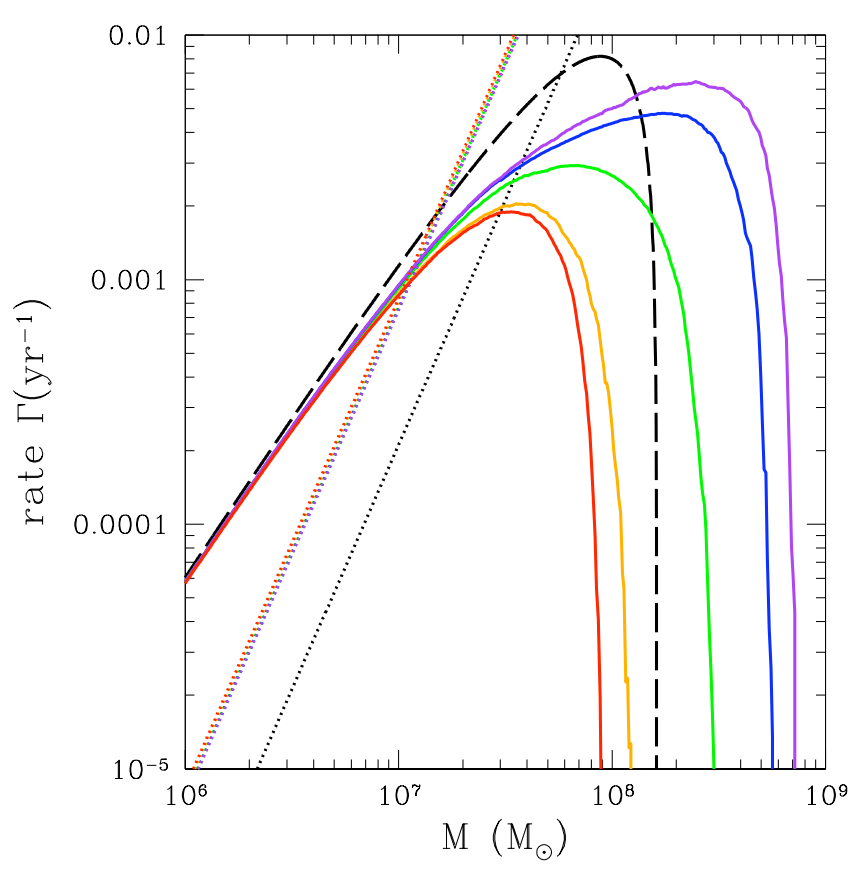

FIG. 3: The rates at which stars are directly captured (dotted lines) and tidally disrupted (solid and dashed curves) by SBHs of mass $M$ in constant-density cores with $n=10^{5} \mathrm{pc}^{-3}$ and $\sigma=100 \mathrm{~km} / \mathrm{s}$. The black curves show the Newtonian rates of Eqs. 21] and 23, while the colored curves show the relativistic rates for SBHs with spins $a / M=0$ (red), 0.5 (orange), 0.9 (green), 0.99 (blue), and 0.999 (purple). The capture rates mildly decrease with SBH spin, while for $M \geq 10^{8} M_{\odot}$ the TDE rates greatly increase with $\mathrm{SBH}$ spin.

the fraction $F_{\text {cap }}$ of simulated geodesics that cross the event horizon. The TDE rate $\Gamma_{\mathrm{TD}}$ is similarly found by multiplying $\Gamma_{\text {tot }}$ by the fraction $F_{\mathrm{TD}}$ of orbits that violate the relativistic criterion f11 for tidal disruption. If $r$ and $\Theta_{\max }$ are chosen large enough, these fractions $F \propto\left(r \sin ^{2} \Theta_{\max }\right)^{-1}$ so that the physical rates are independent of our choice of initial conditions.

In Fig. 3, we show the direct-capture rate $\Gamma_{\text {cap }}$ and TDE rate $\Gamma_{\text {TD }}$ as functions of SBH mass $M$ for our fiducial choices $n=10^{5} \mathrm{pc}^{-3}$ and $\sigma=100 \mathrm{~km} / \mathrm{s}$. The Newtonian prediction for $\Gamma_{\text {cap }}$ underestimates the true relativistic capture rate by about a factor of 4 . If we had used the true specific angular momentum $L_{z}=4 G M / c$ for marginally bound geodesics of a Schwarzschild SBH as the upper limit of the integral in our Newtonian prediction (21), we could have nearly reproduced the correct relativistic result. The capture rate is nearly independent of SBH spin as indicated by the colored dotted lines lying almost on top of each other. This is surprising, since the specific angular momentum $L_{z}$ for prograde (retrograde) marginally bound equatorial orbits varies from $4 M(-4 M)$ to $2 M(-4.828 M)$ as $a / M$ increases from 0 to 1 . Near perfect cancellation over orbital orientation $(\theta, \Theta, \Phi)$ must occur for the capture rate $\Gamma_{\text {cap }}$ to be so mildly dependent on spin, but we do not see any obvious reason for this to be the case. Young et al. 48] calculated the ratio of the capture rate $\Gamma_{\text {cap }}(a)$ for Kerr $\mathrm{SBH}$ of spin $a$ to the capture rate $\Gamma_{\text {cap }}(0)$ for nonspinning SBHs. Equation (B2) of their paper shows that this ratio is approximately given by

$\frac{\Gamma_{\text {cap }}(a)}{\Gamma_{\text {cap }}(0)}=1-0.0820\left(\frac{a}{M}\right)^{2}+0.0717\left(\frac{a}{M}\right)^{4}-0.0864\left(\frac{a}{M}\right)^{6}$.

The small numerical values of the coefficients in this expression indicate the weak dependence of the directcapture rate on $\mathrm{SBH}$ spin; the ratio is between 0.9 and unity over the full range of spins $0 \leq a / M \leq 1$.

The TDE rate $\Gamma_{\mathrm{TD}}$ exhibits a much stronger dependence on SBH spin, as illustrated by the strongly varying solid colored curves in Fig. 3. At small SBH masses, $r_{\mathrm{TD}} \gg M$ and the TDE rate for all spins converges to the Newtonian result as expected. However, as $M$ increases, tidal disruption occurs closer to the SBH where the Newtonian approximation is increasingly invalid. This is most glaringly apparent for masses $M \gtrsim M_{\max }$ of Eq. (1) for which tidal disruption would not be possible in the Newtonian limit. The true maximum mass, where the solid colored curves in Fig. 3 intersect $\Gamma_{\mathrm{TD}}=0$, is given as a function of spin in Fig. 1. Since $\Gamma_{\mathrm{TD}}^{\prime} \propto M^{4 / 3}$, these massive SBHs are capable of tidally disrupting even more stars than their less massive counterparts. Although the spins $a / M=0.99$ and 0.999 depicted by the blue and purple curves in Fig. 3 may seem extreme, the simple scenario of growing a SBH from a standard thin accretion disk leads to a limiting spin $a / m \simeq 0.998$ quite close to the purple curve [29. Although uncertain, cosmological predictions for SBH spin distributions can also be peaked near these large values [31. The primary conclusions to draw from our analysis are that relativistic corrections to the TDE rate can alter predictions by a factor of several for $M \gtrsim 10^{7} M_{\odot}$, and that they can allow TDEs to occur for SBHs as large as $\sim 10^{9} M_{\odot}$.

\section{B. Real galaxies}

Following Frank and Rees 9, the rates we calculated in the preceding subsection assumed that galaxies had constant-density cores outside the SBH's radius of influence,

$$
r_{h} \equiv \frac{G M}{\sigma^{2}}=0.43 \mathrm{pc}\left(\frac{M}{10^{6} M_{\odot}}\right)\left(\frac{\sigma}{100 \mathrm{~km} / \mathrm{s}}\right)^{-2} .
$$

Real galaxies with either power-law or core profiles have mass-density profiles $\rho(r)$ that monotonically decrease with radius. This raises the question of what is the appropriate number density $n$ to insert in our expressions for direct-capture and TDE rates. Frank and Rees [9] argued that the appropriate density to use is that at the critical radius $r_{\text {crit }}$ at which stellar diffusion can refill the loss cone of tidally disrupted orbits on a dynamical time. 
A very crude estimate of this density can be made by assuming that $r_{\text {crit }} \simeq r_{h}$, an assumption roughly true for real galaxies as indicated by Fig. 6 of Wang and Merritt 22. If we further assume that the density profile of galactic centers is that of a single isothermal sphere,

$$
\rho(r)=\frac{\sigma^{2}}{2 \pi G r^{2}},
$$

then inserting $n=\rho\left(r_{h}\right) / m_{*}$ into Eq. (23) implies

$$
\Gamma_{\mathrm{TD}}^{\prime} \simeq 1.3 \times 10^{-3} \mathrm{yr}^{-1}\left(\frac{M}{10^{6} M_{\odot}}\right)^{-2 / 3}\left(\frac{\sigma}{100 \mathrm{~km} / \mathrm{s}}\right)^{5} .
$$

Wang and Merritt [22] use the isotropic distribution function appropriate for a single isothermal sphere to calculate the true rate at which the loss cone is refilled by stellar diffusion. They find that their results are well approximated by the fit

$$
\Gamma_{\mathrm{TD}}^{\prime} \simeq 2.5 \times 10^{-3} \mathrm{yr}^{-1}\left(\frac{M}{10^{6} M_{\odot}}\right)^{-1}\left(\frac{\sigma}{100 \mathrm{~km} / \mathrm{s}}\right)^{7 / 2} .
$$

If we combine this estimate with a recent determination of the relation between SBH mass and host-galaxy velocity dispersion [47,

$$
\frac{M}{10^{6} M_{\odot}}=7.58\left(\frac{\sigma}{100 \mathrm{~km} / \mathrm{s}}\right)^{4.32},
$$

we arrive at a final TDE rate of

$$
\Gamma_{\mathrm{TD}}^{\prime} \simeq 4.8 \times 10^{-4} \mathrm{yr}^{-1}\left(\frac{M}{10^{6} M_{\odot}}\right)^{-0.19}
$$

in the Newtonian limit. This estimate should be reasonable for the power-law galaxies that dominate the total TDE rate; the core galaxies that host the most massive SBHs have TDE rates $\Gamma_{\mathrm{TD}}^{\prime} \simeq 10^{-5} \mathrm{yr}^{-1}$ about an order of magnitude below that of comparable-mass power-law galaxies 22.

In Fig. 4. we show how the direct capture of stars by spinning SBHs changes this prediction. This figure was prepared with the same set of Monte Carlo simulations described in Sec. III. Although there are considerable differences between the Newtonian predictions of Eqs. (23) and (31), these differences result from different treatments of the stellar populations far from the SBH. We may therefore simply renormalize our relativistic predictions $\Gamma_{\mathrm{TD}}=F_{\mathrm{TD}} \Gamma_{\text {tot }}$ of the previous subsection by dividing by Eq. (23) and multiplying by Eq. (31) at each SBH mass $M$. Direct capture reduces the predicted TDE rate by a factor $\sim 2 / 3(1 / 10)$ at $M=10^{7}\left(10^{8}\right) M_{\odot}$. Although TDEs are very rare for large SBH masses, they are still possible for $M<M_{\max } \simeq 10^{9} M_{\odot}$. Since SBHs with masses $M \simeq 10^{9} M_{\odot}$ predominantly live in galaxies with cored profiles, Fig. 4 may somewhat underestimate TDE rates at these masses since the stellar density will not fall as steeply with $r$ as the single isothermal profile of Eq. 27).

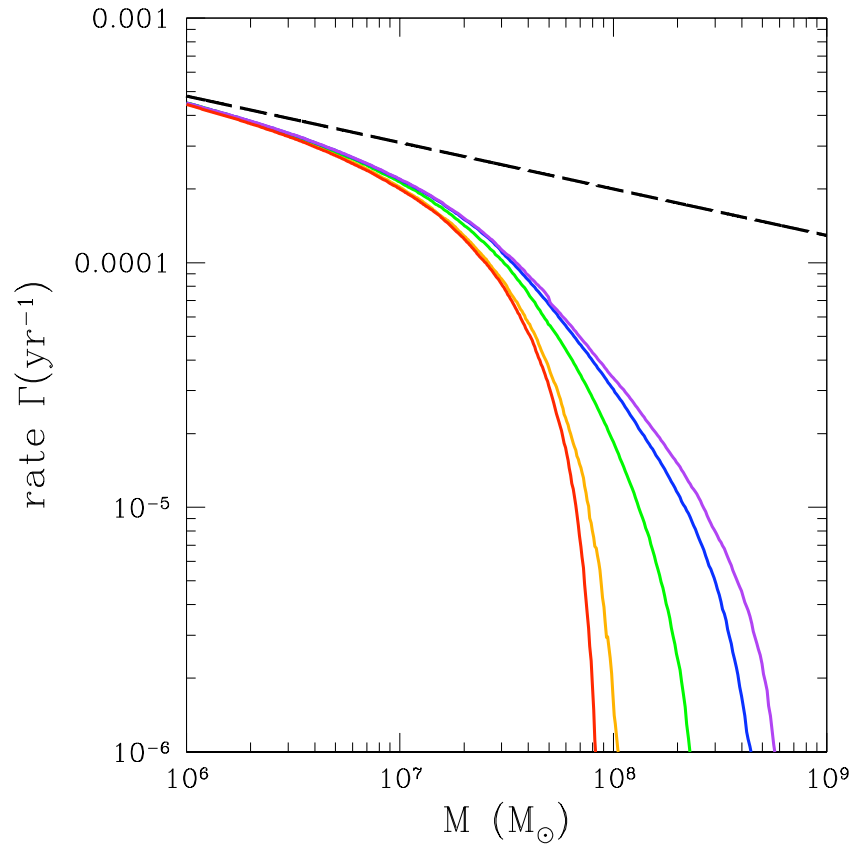

FIG. 4: The rates at which stars are tidally disrupted by SBHs of mass $M$ in power-law galaxies obeying the $M-\sigma$ relation. The dashed black line is the prediction of Wang and Merritt 22 for $\Gamma_{\mathrm{TD}}^{\prime}$ with an updated $M-\sigma$ relation. The colored curves show our relativistic corrections $\Gamma_{\mathrm{TD}}$ to this prediction. The TDE rate increases with $\mathrm{SBH}$ spin, with the given curves corresponding to $a / M=0$ (red), 0.5 (orange), 0.9 (green), 0.99 (blue), and 0.999 (purple).

\section{DISCUSSION}

Astronomers have sought to observe the electromagnetic flares associated with TDEs ever since this possibility was proposed by Rees [10]. Several potential TDEs were discovered over the past 15 years by the Roentgen Satellite (ROSAT) 12 and the Galaxy Evolution Explorer [13, and the recent discovery of additional TDEs by both the Sloan Digital Sky Survey [14] and Swift [15] 18. has renewed interest in this phenomenon. While individual TDEs may provide new insights into SBH accretion physics, the large samples that may soon be available [14] will uniquely probe the whole population of both active and quiescent SBHs. While overall TDE rates depend on stellar populations at galactic centers, the upper bound on the mass $M$ of SBHs capable of tidal disruption is a sensitive measure of SBH spins. For $M \gtrsim 10^{7} M_{\odot}$, tidal disruption occurs deep enough in the SBHs potential well that Newtonian gravity is no longer valid. Furthermore, there is no reason to expect the orbital angular momenta of tidally disrupted stars to align with SBH spins. For both these reasons, accurate calculations of TDE rates require evaluation of the relativistic tidal tensor $C_{i j}$ on a representative sample of generically oriented Kerr geodesics. 
We have performed a series of Monte Carlo simulations that provide this required sample. We use this sample to calculate TDE rates for spinning $\mathrm{SBH}$ as a function of their mass $M$, both in constant-density cores and in isothermal spheres that approximate real powerlaw galaxies. We find that for $M \gtrsim 10^{7} M_{\odot}$, a significant fraction of stars will be directly captured by the SBH's event horizon instead of being tidally disrupted and subsequently accreted. This will reduce the observed TDE rate assuming that directly captured stellar debris will not have the chance to radiate appreciably before being swallowed by the $\mathrm{SBH}$. Above $M \simeq 10^{8} M_{\odot}$, only highly spinning $(a / M \gtrsim 0.9) \mathrm{SBHs}$ will be able to produce observable TDEs. Theory [31] and observation [32, 33] suggest that most SBHs may have such large spins, but further observations are needed to investigate this possibility. A future survey like the Large Synoptic Survey Telescope [19] that finds thousands of TDEs may provide important constraints on the distribution of SBH spins.

Acknowledgements. We would like to thank Mike Blanton, Glennys Farrar, Andrei Gruzinov, David Merritt, Maryam Modjaz, Sterl Phinney, and Scott Tremaine for useful conversations.
[1] C. Seyfert, Astrophys. J. 97, 28 (1943).

[2] F. Hoyle, W. A. Fowler, Mon. Not. Roy. Astron. Soc. 125, 169 (1963).

[3] D. Lynden-Bell, Nature 223, 690 (1969).

[4] J. Kormendy, D. Richstone, Ann. Rev. Astron. Astrophys. 33, 581 (1995).

[5] J. Magorrian, S. Tremaine, D. Richstone, R. Bender, G. Bower, A. Dressler, S. M. Faber, K. Gebhardt et al., Astron. J. 115, 2285 (1998).

[6] K. Gebhardt, R. Bender, G. Bower, A. Dressler, S. M. Faber, A. V. Filippenko, R. Green, C. Grillmair et al., Astrophys. J. 539, L13 (2000).

[7] A. Toomre, J. Toomre, Astrophys. J. 178, 623 (1972).

[8] J. E. Barnes, L. E. Hernquist, Astrophys. J. 370, L65 (1991).

[9] J. Frank, M. J. Rees, Mon. Not. Roy. Astron. Soc. 176, 633 (1976).

[10] M. J. Rees, Nature 333, 523 (1988).

[11] L. E. Strubbe, E. Quataert, Mon. Not. Roy. Astron. Soc. 415, 168 (2011).

[12] N. Bade, S. Komossa, M. Dahlem, Astron. Astrophys. 309, L35 (1996).

[13] S. Gezari, D. C. Martin, B. Milliard, S. Basa, J. P. Halpern, K. Forster, P. G. Friedman, P. Morrissey et al., Astrophys. J. 653, L25 (2006).

[14] S. van Velzen, G. R. Farrar, S. Gezari, N. Morrell, D. Zaritsky, L. Ostman, M. Smith, J. Gelfand, A. J. Drake, arXiv:1009.1627 [astro-ph.CO]].

[15] D. N. Burrows, J. A. Kennea, G. Ghisellini, V. Mangano, B. Zhang, K. L. Page, M. Eracleous, P. Romano et al., Nature 476, 421 (2011).

[16] A. J. Levan, N. R. Tanvir, S. B. Cenko, D. A. Perley, K. Wiersema, J. S. Bloom, A. S. Fruchter, A. d. U. Postigo et al., Science 333, 199 (2011).

[17] J. S. Bloom, D. Giannios, B. D. Metzger, S. B. Cenko, D. A. Perley, N. R. Butler, N. R. Tanvir, A. J. Levan et al., Science 333, 203 (2011).

[18] S. B. Cenko, H. A. Krimm, A. Horesh, A. Rau, D. A. Frail, J. A. Kennea, A. J. Levan, S. T. Holland et al., arXiv:1107.5307 [astro-ph.HE]].

[19] P. A. Abell et al. [ LSST Science and LSST Project Collaborations ], arXiv:0912.0201 [astro-ph.IM]].

[20] L. Spitzer and R. Harm, Astrophys. J. 127, 544 (1958).

[21] H. Cohn and R. Kulsrud, Astrophys. J. 226, 1087 (1978).

[22] J. -X. Wang, D. Merritt, Astrophys. J. 600, 149 (2004).

[23] F. K. Manasse and C. W. Misner, J. Math. Phys. 4, 735
(1963).

[24] J.-A. Marck, Proc. R. Soc. Lond. A 385, 431 (1983).

[25] R. P. Kerr, Phys. Rev. Lett. 11, 237 (1963).

[26] A. M. Beloborodov, A. F. Illarionov, P. B. Ivanov, A. G. Polnarev Mon. Not. Roy. Astron. Soc. 259, 209 (1992).

[27] P. B. Ivanov, M. A. Chernyakova, Astron. Astrophys. 448, 843 (2006).

[28] N. I. Shakura and R. A. Sunyaev, Astron. Astrophys. 24, 337 (1973).

[29] K. S. Thorne, Astrophys. J. 191, 507 (1974).

[30] J. M. Bardeen, Nature 226, 64 (1970).

[31] E. Berti, M. Volonteri, Astrophys. J. 684, 822 (2008).

[32] C. S. Reynolds, A. J. Young, M. C. Begelman and A. C. Fabian, Astrophys. J. 514, 164 (1999).

[33] L. W. Brenneman and C. S. Reynolds, Astrophys. J. 652, 1028 (2006).

[34] R. H. Boyer and R. W. Lindquist, J. Math. Phys. 8, 265 (1967).

[35] B. Carter, Phys. Rev. 174, 1559 (1968).

[36] M. Ishii, M. Shibata, Y. Mino, Phys. Rev. D 71, 044017 (2005).

[37] C. D. Murray, S. F. Dermott, Solar System Dynamics (Cambridge University, New York, 1999).

[38] E. S. Phinney, in The Center of the Galaxy: Proceedings of the 136th Symposium of the International Astronomical Union, edited by M. Morris (Kluwer Academic Publishers, Dordrecht, 1989), p. 543.

[39] J. M. Bardeen, W. H. Press, S. A. Teukolsky, Astrophys. J. 178, 347 (1972).

[40] H. Sponholz, Mem. Soc. Astron. It. 65, 1135 (1994).

[41] S. Kobayashi, P. Laguna, E. S. Phinney, P. Meszaros, Astrophys. J. 615, 855 (2004).

[42] J. H. Krolik, T. Piran, arXiv:1106.0923 [astro-ph.HE]].

[43] S. M. Faber, S. Tremaine, E. A. Ajhar, Y. -I. Byun, A. Dressler, K. Gebhardt, C. Grillmair, J. Kormendy et al., Astron. J. 114, 1771 (1997).

[44] R. M. Buchholz, R. Schodel, A. Eckart, Astron. Astrophys. 499, 483 (2009).

[45] J. N. Bahcall, R. A. Wolf, Astrophys. J. 209, 214 (1976).

[46] D. Merritt, Astrophys. J. 718, 739 (2010).

[47] A. Schulze, K. Gebhardt, Astrophys. J. 729, 21 (2011).

[48] P. J. Young, G. A. Shields, J. C. Wheeler Astrophys. J. 212, 367 (1977). 